\title{
Science of the Vatican Observatory since 2000
}

\author{
Guy Consolmagno, S.J. \\ The Vatican Observatory \\ gjc@specola.va
}

\begin{abstract}
Five research areas have been the focus of the scientific work of the Specola Vaticana (Vatican Observatory) over the past twenty years: planetary sciences, stellar astronomy, extragalactic astronomy, cosmology, and the development of the Vatican Advanced Technology Telescope (VATT). The choice of research program is left to the individual astronomers, all of whom work closely with lay collaborators around the world. Notable, especially in connection with the VATT, is the close coordination of the Specola with the Steward Observatory of the University of Arizona. One unique strength of the Specola is its independence from short-term funding requirements. As a result of its stable funding, Specola astronomers can engage in longterm research programs such as surveys of meteorite properties, exoplanets, stellar clusters, and galaxy clusters, which may take ten or more years to come to fruition. In this way the Specola complements the large research programs of contemporary astronomy.
\end{abstract}

\section{Keywords}

Specola Vaticana (Vatican Observatory) - planetary sciences - stellar astronomy extragalactic astronomy - Vatican Advanced Technology Telescope (VATT)

\section{Introduction}

The Specola Vaticana - the Vatican Observatory-(Specola) is one of the oldest astronomical research institutions in the world. In one sense, it can be dated back to the reform of the calendar in 1582 , when the pope first consulted astronomers at the Roman College, notably the Jesuit Christopher Clavius

(C) GUY CONSOLMAGNO, S.J., 2020 | DOI:10.1163/22141332-00702008

This is an open access article distributed under the terms of the prevailing CC-BY-NC-ND 4.0 license. 
(1537-1612). It has existed in its current form since its institution by Pope Leo XIII (r.1878-1903) in 1891. In 1906, the first Jesuit director, Johann G. Hagen (1847-1930), was named. Since 1935, it has had its headquarters at the papal summer residence in Castel Gandolfo outside Rome; at that time, the staffing of the observatory was given over to the Jesuit order, an arrangement that continues to the present day.

In 1980, in order to flee the light pollution around Rome, a dependent research center was opened at the Steward Observatory of the University of Arizona in Tucson, USA. The Vatican Observatory Research Group (VORG) now operates the 1.8m Alice P. Lennon Telescope with its Thomas J. Bannan Astrophysics Facility, known together as the Vatican Advanced Technology Telescope (VATT), located at the Mount Graham International Observatory (MGIO) in southeastern Arizona. The VATT itself is funded by the Vatican Observatory Foundation (Vof).

A detailed history of the Specola can be found in Fr. Sabino Maffeo's books: Nove papi, una missione: Cento anni della Specola Vaticana (Vatican City: Specola Vaticana, 1991), La Specola Vaticana: Nove papi, una missione (Vatican City: Pubblicazioni della Specola Vaticana, 2001) (In the Service of Nine Popes: 100 Years of the Vatican Observatory, trans. George V. Coyne, S.J. [Vatican City: Specola Vaticana, 1991]), and The Vatican Observatory: In the Service of Nine Popes, trans. George Coyne (Notre Dame, IN: Notre Dame Press, 2001). A condensed version of that history appeared as a chapter of the book: Guy Consolmagno, The Heavens Proclaim: Astronomy and the Vatican (Huntington, IN: Our Sunday Visitor Press, 2009), published in celebration of the International Year of Astronomy honoring the four hundredth anniversary of Galileo's (1564-1642) telescope. That chapter is available for download from the Vatican Observatory Foundation website, https://www.vofoundation.org/faith-and-science/history-vatican-observatory/. However, the most recent edition of Maffeo's book is now nearly twenty years old, and even the updated chapter in The Heavens Proclaim is a decade out of date. Thus, I wish here to describe the scientific work of the Specola in recent years.

I outline five research areas that have been the focus of the scientific work of the Specola over the past twenty years: planetary sciences, stellar astronomy, extragalactic astronomy, cosmology, and the VATT itself. Note that in addition to these topics, members and affiliates of the Specola are also very active in the scholarly study of the history and philosophy of science, on issues in science and theology, and in education and public engagement in science and of science and faith. Those topics, however, are beyond the scope of this paper, and deserve a treatment at least as extensive as this one. 


\subsection{Overview}

The work of the planetary sciences group at the Vatican Observatory is primarily centered on the nature of small bodies within our own solar system and the discovery of planets around other stars. The modern study of planets as places of interest began with the pioneering work of Angelo Secchi, S.J. (1818-78) in the mid-nineteenth century. His 1859 book, Quadro fisico del sistema solare (The physical framework of the solar system) (Rome: Tipografia delle Belle Arti), was perhaps the first work ever to treat planets not as mere dots of light whose orbits were to be calculated, but as places worthy of scientific study in and of themselves.

In the middle of the twentieth century, a number of Specola astronomers were involved in the field. For example, in 1946, Br. Mattieu Timmers, S.J. $(1907-54)$ gained note in the astronomical world for the discovery of a comet, while for many years Ernst Salpeter, S.J. (1912-76) served as the curator of the meteorites donated to the Vatican from the collection of Adrien Charles, marquis of Mauroy (1848-1927). However, with the death of Salpeter in 1976, this work was left in abeyance.

\subsection{Meteoritics}

Br. Guy Consolmagno, S.J. arrived at the Specola in 1993. In 1996, he began a collaboration with Daniel Britt, now at the University of Central Florida (UCF), to survey the physical properties of meteorites - particularly density and porosity, along with thermal and magnetic properties. The survey of meteorite density began with measurements of the extensive Vatican collection. In 2004, Br. Robert Macke, S.J. began working as an external collaborator on this project and this work became a part of his doctoral program under Britt at UCF. He joined the Specola staff full time in 2013, at which point he took over from Consolmagno the duties of meteorite curator.

Macke has further applied the techniques for measuring meteorite physical properties, developed at the Specola and UCF, to measure the physical properties of lunar samples (including Apollo samples) and Martian meteorites, applying these to our understanding of the nature of the Moon and Mars. This work involves travel to measure samples in museums, universities, and private collections in the US and Europe plus travel to the repository of Apollo samples and Antarctic meteorites at the NASA Johnson Space Center in Houston, Texas. From this, he has become a key figure in an international program of organizing and systematizing how meteorites are curated. 
The most recent systematic measurements of meteorite properties have been the collection of meteorite heat capacity data. This work is being carried out using both facilities at Boston College, in collaboration with Cyril Opeil, S.J., and using a unique liquid-nitrogen based system in the meteorite laboratory in Castel Gandolfo.

Macke is also participating in three NASA-funded initiatives with Britt at UCF. One is a continuation of the grant that supported Macke's thesis work, which funds further travel and expenses for continued measurement of meteorite physical properties in collections around the world. Another is a NASAsupported Solar System Exploration Research Virtual Institute, the Center for Lunar and Asteroid Surface Science, which provides the context in which the meteorite lab measurements are integrated into the larger context of understanding asteroid origins, evolution, and current states. Finally, Macke has been named as a participating scientist in the NASA-funded LUCY mission to send a spacecraft to an asteroid in the Trojan belt, co-orbiting with Jupiter.

These meteorite physical properties have been applied to derive a more general picture of the physical state of the asteroid belt, how the densities of asteroids vary with distance from the Sun and what that might tell us about planetary formation and early evolution. In connection with this work, from 2012 to 2015, Consolmagno participated in "Vesta, the Key to the Origins of the Solar System," an international team led by Diego Turrini (INAF-IAPS) through the International Space Science Institute (ISSI) in Bern, Switzerland. His knowledge of basaltic achondrite meteorites evolution and densities allowed the team to constrain the evolution of the differentiated asteroid 4 Vesta, visited by the NASA Dawn spacecraft from 2011 to 2012.

Lastly is the continuing important work in meteorite curation and loans. As one of the most accessible sources of meteorite material for research, the curation activities of the meteorite laboratory represent an important contribution to meteorite and planetary sciences. For example, more than half of the papers published since 2000 analyzing the unique Martian meteorite Chassigny have been based on thin sections provided from the Vatican Observatory collection. Furthermore, in his work surveying meteorite and lunar sample properties, Macke traveled to many of the major meteorite collections around the world; in the process of these trips, he had the remarkable experience of handling thousands of different extraterrestrial samples and seeing how they are curated worldwide. As a result, he has become instrumental in organizing the community of meteorite curators. In 2018, Macke chaired the first international workshop on meteorite curation at the Specola headquarters in Castel Gandolfo. 


\subsection{Meteors}

Jean Baptiste Kikwaya Eluo, S.J. became affiliated with the Specola in 2003, earning his $\mathrm{PhD}$ under Peter Brown and Margaret Campbell at the University of Western Ontario (WU). As a result of that work, in 2009, he and his advisors published the first reliable estimates of the density of small meteoroids. With collaborators Brown, Campbell, and William J. Cooke at WU he has continued to work on measuring the physical properties of meteors, using this information to determine the different physical and chemical natures of their sources, comets and asteroids.

In 2014, Kikwaya Eluo set up a network of all-sky cameras with the collaboration of the NASA Meteoroid Environment Office (MEO) based at the NASA Marshall Space Flight Center in Huntsville, Alabama, and with active participation from the University of Arizona Lunar and Planetary Lab (LPL), the Planetary Science Institute (PSI), the National Optical Astronomy Observatory, the MмT Observatory of Mount Hopkins, and WU.

The ultimate goal is that, given the record of a fireball that produces meteorites, these cameras can help us find and recover those meteorites. From this, one can study the phenomenon of a meteorite's fall from its entry in the atmosphere to meteorite recovery. In this way, one could compare the chemical and physical properties of the meteoroid, including its bulk density, grain density, heat capacity, and thermal conductivity, as inferred from the bolide light curves and spectra, to the physical characteristics of the recovered meteorite itself. On June 2, 2016, the first meteorite was recovered in central Arizona from a bolide captured by this network.

\subsection{NEO Observations}

Beginning in 2015, Kikwaya Eluo, Specola members Richard P. Boyle and Christopher J. Corbally, and collaborators Carl Hergenrother, Ed Tedesco, and others at PSI and the LPL have worked to develop the ability of the VATT to observe and characterize Near Earth Objects (NEOs). These objects are of interest for a number of reasons: they are the closest asteroids to the Earth, they are potentially hazardous objects, and they are possible targets for future manned and unmanned missions, for both scientific and commercial exploitation.

Current research on NEOs is mostly limited at present to addressing the question of their number and location, in particular with an eye as to how hazardous they may be to the Earth; the physical characteristics of these objects generally remains unknown. For example, since 1998 the Catalina (Arizona) Sky Survey (css), capable of detecting objects as faint as 20.5 magnitude, 
has submitted thousands of astrometric observations to the Minor Planet Center (MPC) resulting in the discovery of more than nine thousand NEOs (as of January 1, 2019). The near-Earth asteroid 101955 Bennu was chosen as the target of NASA's OSIRIS-Rex sample return mission (run by the LPL) in part because its spectrum suggests it is a carbonaceous asteroid. That physical characterization could only have been accomplished through techniques of observations other than merely astrometry.

The major difficulty in characterizing such objects is that they are small, faint, and fast-moving. The time from discovery to when they are no longer able to be observed may be as short as one week. From 2015, the Specola has begun to plan to observe these newly discovered NEOs simultaneously using three different instruments on three Arizona telescopes with current instrumentation: the f/9 Ritchey-Chretien focus at the 9o-inch Bok telescope on Kitt Peak, the 61-inch LPL telescope on Mt. Bigelow, and the VATT. The process of coordinating these telescopes, being carried out by Paul Gabor, S.J. (see below), is now well underway. The first steps in this program have been the procurement by Tedesco at PSI of eight color filters used in the Tholen classification of NEO asteroids. These filters are presently being validated against standard star fields at the VATT.

\subsection{Trans-Neptunian Objects}

Beginning in the mid-199os, Consolmagno has collaborated with Stephen Tegler of Northern Arizona University and William Romanishin of the University of Oklahoma to conduct a long-term survey at the VATT of Trans-Neptunian Objects (TNOs) broad band colors, which characterize their surface composition, and recording their variation of brightness as they spin, which can eventually provide information about their shapes and possible spots on their surfaces. A series of papers by this team has revealed that most TNOs can be grouped into two distinct color populations, red and gray. Further, they found that while TNOs whose orbits have been excited into high inclinations or eccentricities can come in either color, those whose orbits appear undisturbed are almost entirely found in the red population.

In addition, since 2009, Boyle, with collaborators Cernis and Laugalys (Vilnius, Lithuania), has been doing astrometry on asteroids and trans-Neptunian objects. As a result of their observations, over a hundred new asteroids and TNOs have been discovered at the VATT including one that is large enough to be nearly dwarf-planet sized. Following IAU conventions that such objects are to be named for deities related to creation, they have named it Praamzius for the goddess of creation in Lithuanian mythology. 


\section{$2.6 \quad$ Exoplanets}

Gabor joined the Specola staff in 2010, following doctoral work at the Institut d'Astrophysique Spatiale, University of Paris XI, on the detection of planetary systems around other stars. In 2014, he organized a conference in Tucson, Arizona, "Search for Life Beyond the Solar System: Exoplanets, Biosignatures \& Instruments." This conference brought together an interdisciplinary community to examine the interface of exoplanet observations, early and extreme forms of life on Earth, atmospheric biomarkers, and planet-finding telescopes.

Gabor has been instrumental in setting up an optical fiber connection between vatT and the Potsdam Echelle Polarimetric and Spectroscopic Instrument (PEPSI) detector at the Large Binocular Telescope on Mt. Graham. First tested in 2013, this combination of VATT with the PEPSI detector can produce high-resolution spectra to confirm and characterize possible exoplanet systems found via transit by the Kepler and TESS spacecrafts. The uniqueness of the combination of the VATT and PEPSI is that it can be operated for many nights during a year, and thus it samples an orbital period domain not accessible to any other facility in the world.

Gabor and Boyle are also playing key roles in the Exo-Earth Discovery and Exploration Network (EDEN). It aims to examine all M stars within fifty lightyears and conclusively determine whether there are any Earth-sized planets in their habitable zones. The EDEN transit survey is a multinational collaboration, created by Daniel Apai (University of Arizona). EDEN has the capability of carrying out the most sensitive transit survey in the northern hemisphere.

3

\section{Stellar Astronomy}

\subsection{Overview}

Stellar astrophysics is the study of the origin, formation, evolution, and fate of stars and of the mechanisms (either nuclear, atmospheric, or exterior interactions with companions/other objects) by which they work and interact with their stellar environment. This field has made remarkable advances in the last twenty years through a combination of revolutionary advances in computing (theoretical studies and data reduction) and through a plethora of missions and sky surveys. It has also historically been an important component of research at the Specola, dating back to its participation in the international Carte du Ciel survey in 1891. Because the Jesuit astronomers at the Specola are not 
subject to time limits of grants or tenure review, they are uniquely situated to carry out such long-term surveys.

\subsection{Open Cluster Spectrophotometry}

Richard Boyle, S.J. arrived at the Specola in 1981. Since that time, in collaboration with astronomers in Italy, Poland, and especially Lithuania, he has refined a system of specially chosen filters, the Vilnius seven-filter intermediate-band photometric system, to classify stars in stellar clusters, pursuing a number of different projects possible with the accurate photometric classification of stars.

By using this method to classify stars according to temperature, surface gravity, luminosity, and metallicity even in interstellar dust-reddened fields, this team has determined stellar populations, distances, reddening, and total extinction (and metallicities in some cases) for members of open clusters and field stars. Their photometric studies have determined how to distinguish those stars that belong to clusters from stars found that simply happen to lie within the same line of sight in a particular field of view. This allows for a comparison of different clusters in the galaxy, which can lead to a better idea of mass distribution in the galaxy. They have also made similar photometric studies for fields defined for the Kepler space mission, which has been key in the detection of Earth-sized planets.

\subsection{Stellar Spectrophotometry}

Christopher Corbally, S.J. has been a member of the Specola staff since 1984 . Beginning in 2003, he has been a key participant in the Nearby Stars (NStars) project, the spectral analysis of the 3,60o stars within forty parsec of the sun and earlier than Mo spectral type. The goal of this program is to determine new, precise spectral types and basic physical parameters for such objects. These results can be applied to problems ranging from the measure of the chromospheric activity of these stars, to better understanding the basic physical parameters of stars with exoplanets. For example, one result suggests that stars with planets (extrasolar planets) have a higher heavy element abundance than those without planets.

Corbally and collaborators have been studying the spectroscopy of peculiar stars and binaries, including those in the Aries cloud, to determine precise spectral classes, luminosities, and any peculiarities. From this, a distance and extinction study could be completed for star-forming regions of our galaxy. Another spectroscopy project by this group is looking for planetesimal belts around binary systems, by examining debris disks in main sequence 
binary systems using the Multiband Imaging Photometer on the Spitzer Space Telescope. In addition, extensive work is being done on peculiar stars such as the lambda Boötis type and Barium II (Ba) stars, which have yet to be explained by standard theories of stellar formation and evolution. And finally, Corbally has developed an encoded system for classification of stellar spectra that are stored in individual databases.

Corbally is currently participating with researchers from Belgium, China, Italy, Poland, and the United States in a spectroscopic follow-up to exoplanet surveys such as that conducted by Kepler space telescope. The aim is to use spectral ground-based data for these stars, including data from the Chineseadministered 4-m LAMOST telescope, to determine the physical parameters of stars in the Kepler data. Richard Gray (Appalachian State University, Boone, North Carolina) and Corbally complement this effort by obtaining the stars' spectral classifications, as a check on those obtained by LAMOST. Given the high number of stars, they have developed an automatic classification code, MKCLASS, for this task. This code surpasses any previous auto-classification method in accuracy and scope.

\subsection{Stellar Evolution}

David Brown S.J. joined the Specola staff in 2008. Since then, he has been involved in the study of formation channels of hot subdwarf stars in both the galactic field and globular clusters. In recent years, surveys like Kepler have yielded a wealth of information, especially through asteroseismology. Brown has used theoretical modeling using stellar evolution codes and population synthesis programs to try to understand the exact formation mechanisms of these stars, producing models that can be tested in part by the results of those surveys.

These hot subdwarf stars are very intricately linked with a number of issues in astrophysics. These include the second parameter problem and multiple stellar population puzzles in globular clusters, binary evolution via Roche-lobe overflow, common envelope evolution, and white dwarf mergers. On a galactic scale understanding these stars is key to explaining the ultraviolet upturn phenomenon in giant elliptical galaxies and bulges in spiral galaxies, concerning which the GALEX mission has yielded many useful results.

Meanwhile, using the high-speed Galway UltraFast Imager (GUFI) camera on the VATT, Boyle and his collaborators have determined the optical periodicity in ultracool radio pulsating dwarfs. Similarly, they have participated at the VATT in a large multi-wavelength campaign to investigate anomalous radio emissions in brown dwarfs, which has shed light on the mechanism producing these radio emissions and established their association with the presence of magnetic spots on the surface of brown dwarfs. 


\subsection{Overview}

Galaxies are the crossroads of astronomy: they look up to cosmology and they look down to the interstellar medium and star formation. Galaxies evolve according to the initial and boundary conditions given by cosmology; nearby galaxies are the best laboratories to test a "nearby cosmology." The study of galaxies is a crucial element in connecting our knowledge of the universe as a whole with the formation of stars and planets.

Since 2000, the Vatican Observatory staff has been involved in this area through both the research itself and in the organization of conferences and schools, notably in the work of José Funes, S.J., Rev. Alessandro Omizzolo, and Richard D'Souza, S.J. Funes joined the Specola in 2000 and served as its director for two terms, from 2006 until his return to Argentina in 2015. Omizzolo, of the diocese of Padua, the sole non-Jesuit among the full-time astronomical staff, began his collaboration with the Specola in 1997. And D'Souza became a member of the Specola in 2016, upon the completion of his doctoral studies at the Max Planck Institute in Garching, Germany.

In 2000 and 2007, Funes and his colleagues organized two international conferences on the formation and evolution of disk galaxies; the proceedings were published by the Astronomical Society of the Pacific Conferences Series. Omizzolo and his colleagues organized a workshop on Active Galactic Nuclei (AGN) and galaxy evolution. Funes and colleagues also organized a Vatican Observatory Summer School (voss) on galaxy evolution in 2003, while the 2014 Vatican Observatory Summer School (voss) was dedicated to the comparison of near and far galaxies.

\subsection{Galaxy Evolution}

Our knowledge of galaxy evolution showcases our understanding of cosmology, stellar evolution, and galaxy dynamics. It is an excellent example of how scientific knowledge achieved independently can be put together to shed light on a complex process that involves other physical processes at different scales. But like the galaxies themselves, our scientific understanding of galaxy evolution is still evolving.

From his arrival at the Specola in 2000 until 2015, Funes primarily worked to characterize nearby galaxies. His VATT observations produced broad color (UBV) images of spiral and irregular galaxies (mostly dwarf galaxies) within a distance of $11 \mathrm{Mpc}$. Combined with $\mathrm{H}$-alpha, R and UV imaging from "11HUGs GALEX" (the $11 \mathrm{Mpc}$ H-alpha UV Galaxy Survey, a legacy project of the Galaxy Evolution Explorer), these data helped provide a comprehensive multiwavelength image atlas of the local universe. In addition, his observations at 
the VATT of the ionized gas and the stellar populations in elliptical galaxies with dust lanes shed light on star formation rates and how they have changed over time, advancing the study the environment of these galaxies. His observations also explored the connection between Active Galactic Nuclei and starbursts in these galaxies.

\subsection{Galaxy Clusters}

Galaxies are not exactly "island universes"; they do not evolve in isolation. Galaxies evolve due to the interaction with other galaxies. Spiral galaxies tend to collect in groups of galaxies, which contain up to several dozen galaxies. Elliptical galaxies are more common in clusters of galaxies. Since 2005, Omizzolo has studied galaxy clusters as a member of WINGS (WIde-field Nearby Galaxy-cluster Survey).

WINGS is an all-sky survey of a complete, $X$-ray selected sample of galaxy clusters in the redshift range 0.04-0.07. The goal of the wINGS project is the systematic study of the local cosmic variance of the cluster population and of the properties of cluster galaxies as a function of cluster properties and local environment. This collection of data allows the definition of a local, "zeropoint" reference against which more distant clusters can be compared, allowing us to better gauge cosmic evolution.

With these observations, WINGS has provided photometric data for huge samples of galaxies $(\sim 550,000)$ and stars $(\sim 190,000)$ in the inner field $\left(34^{\prime} \times 34^{\prime}\right)$ of seventy-seven nearby galaxy clusters, as well as structural and morphological information for a sub-sample $\left(\sim 5^{0}, 000\right)$ of relatively bright galaxies. Spectroscopic information for $\sim 6,000$ galaxies in forty-eight WINGS clusters is provided by a follow-up multi-fiber, medium-resolution survey, while additional photometric information comes from follow-up imaging surveys in the near-infrared (NIR) (J,K; 28 WINGS clusters) and U-band (18 WINGS clusters).

The study of dynamics and star formation rates in galaxy clusters can give noteworthy contribution to solve the problem of dark matter. The wINGs team addresses this question with two instruments: wide field imaging of the clusters with the largest possible number of filters (at present the clusters have been surveyed in the U, B, V, NIR, X bands but a new survey is starting with the capability to observe through fifty-six filters) and fiber and long-slit spectroscopy of the cluster galaxies. Using the wide field imaging (field of view from 30' to $90^{\prime}$ ) deep images of every cluster have been obtained, providing aperture and surface photometry and automatic classification of the galaxies. Both the center and the outer parts of each cluster have been observed; the study of the outer parts of the cluster allows one to understand the behavior of the galaxies falling into the cluster and, in particular, what happens to the star 
formation rate in these infalling galaxies (distortion of the galaxies, stripping of the gas, etc.).

Among all the peculiar galaxies, one class has aroused and continues to arouse the interest of that research group are "jellyfish galaxies," so called because the passage of a smaller galaxy through the plane of a larger one leaves behind a string of faint stars reminiscent of the tendrils of jellyfish. In the mid2010s, Omizzolo and his collaborators compiled and published a first catalog of these jellyfish galaxies present in the clusters of the WINGS sample. Their current work is to extend the study of these galaxies to other samples of galaxy clusters, and not limited only to neighboring clusters but also to those more distant.

D'Souza and his collaborators are working on semi-analytic galaxy formation models to study galactic accretion and the outer structure of galaxies in the cold dark matter cosmology. The simulations cover scales from the stellar haloes of Milky Way-like galaxies to the $\mathrm{cD}$ galaxies (galaxies that can be found near the centers of some rich galaxy clusters) and resolve low surface brightness substructure such as tidal streams.

In 2018, D'Souza and Eric Bell (University of Michigan) deduced that the Andromeda galaxy, our closest large galactic neighbor, shredded and cannibalized a massive galaxy two billion years ago. This disrupted galaxy was the third-largest member of the local group of galaxies, after the Milky Way and Andromeda galaxies. Even though it was mostly shredded, this massive galaxy left behind a rich trail of evidence: an almost invisible halo of stars larger than the Andromeda galaxy itself, an elusive stream of stars and a separate enigmatic compact galaxy, M32. This disrupted galaxy was a long-lost sibling of the Milky Way and was nearly half its size in mass. This discovery will help astronomers understand how disk galaxies like the Milky Way evolve and survive large mergers.

\section{Cosmology}

\subsection{Overview}

Over the past seventy-five years, cosmology has come into its own as an extensive sub-discipline of astronomy and physics. It embraces an extremely large and diverse range of questions, from those of recent and early galaxy formation and evolution, and the detailed structure of the later universe and the 
important roles of dark matter and dark energy, to the detailed description of the early universe-inflation and reheating, primordial nucleosynthesis, recombination and the Cosmic microwave Background Radiation (CBR), and the effects of re-ionization with the advent of the first stars and galaxies. Cosmologically oriented astronomers and physicists have made notable strides in unraveling the details of cosmic history from the Big Bang (and perhaps before) to the present by a combination of observational, theoretical, and computational efforts.

The formulation and observational support of the concordance cosmological model of the universe, relying on the incredible results from свR acoustic-peak, baryon acoustic oscillation, and Supernovae Type 1a (SNia) distance-determination data, have provided for the first time a reliable detailed description of the universe and its history back to just after the Planck era. But a great deal remains to be done, both in further confirming and testing this concordance model through further observational and theoretical work and in shedding more light on the mysteries of early and later galaxy evolution and developing a compelling and adequate model of the Planck era (quantum cosmology). The Specola has been involved in this cosmological research over the past thirty years through the theoretical work of the late William Stoeger, S.J. (1943-2014) and of Gabriele Gionti, S.J.

Stoeger arrived at the Specola in 1979 and worked closely with collaborators in South Africa and in Brazil, until his death in 2014. Most of that work centered on the standard isotropic and the spatially homogeneous Friedmann-LemaitreRobertson-Walker (FLRW) zeroth-order model. The goal was to develop a mathematical framework in which cosmological models closely related to, but much more general than, FLRW can be fit to presently available data. Another such effort was to fit data from new, more precise independent observables, such as red-shift drift and the maximum of the angular-diameter distance and its redshift. Stoeger and his collaborators (particularly George Ellis, University of Cape Town, South Africa) also did related cosmological work clarifying certain conceptual issues related to inflationary models, multiverses, and in estimating the boundary between local and cosmological gravitational influences.

Gionti worked with the Specola during his period of regency (2004-6) and joined the Specola full time in 2010. Upon arrival, he investigated key issues in super-string theory, concentrating on the phenomenon of T-duality. These results were oriented toward eventually developing an adequate description of the universe during the Planck era-before the Big Bang — when all the physics we know has broken down and where we must replace it with a complete quantum-gravitational description of reality. 
At the end of 2015, Gionti moved from string theory to studying the asymptotic safety approach to quantum gravity. This attempts to find the quantum mechanical behavior of general relativity using the hypothesis that the infinities of the quantization of gravity can be resolved posing a cut-off on the value of the possible energies (infrared cut-off) and later sending this cut-off to infinity, hoping that the physical quantities do not diverge.

\subsection{Background}

Much of the work described above has involved observations at the Vatican Advanced Technology Telescope (VATT). It is thus worthwhile to describe in detail the history and current capabilities of the VATT and outline how its instrumentation has developed and continues to develop over time.

The Specola originally opened an office at the University of Arizona solely with the idea of taking advantage of the large number of telescopes in the area accessible by astronomers affiliated with the university. The idea that the Specola would be a participant with its own telescope first began in March 1985, when a large mirror was spun-cast by what was to become the Steward Observatory Mirror Laboratory (SOML). This mirror, a 1.8-m diameter dish with an $\mathrm{f} / 1 . \mathrm{o}$ focus, was offered to the Vatican Observatory to be the heart of a joint new telescope project with Steward Observatory.

Once the decision was made to build a telescope around this mirror, the history of the VATT began. In the fall of 1986, the Vatican Observatory Foundation was incorporated and the design of the telescope began. In February 1990, the telescope mount was delivered by L\&F Industries to a test facility near Tucson. In the fall of 1990, the facility's contractor, T.L. Roof, began site clearing on Mt. Graham. In November 1991, polishing of the primary mirror was completed by the SOML. In May 1992, polishing of the secondary mirror was completed by the Space Optics Research Laboratory. In October 1992, the telescope mount and dome were installed on Mount Graham. In September 1993, first light was obtained with the primary mirror; this was followed by the telescope's dedication in September of that year. July 1994 saw first light with the secondary mirror. In January 1995, scientific observations began.

During the years immediately following 1995, the principal effort of the technical team went into further tuning and stabilization of the telescope's very complex systems. Facility instrumentation was limited to CCD cameras, first one on loan from Columbia University and later a $2 \mathrm{~K}$ camera from 
Steward's Imaging Technology Laboratory (ITL). The scientific capabilities were enhanced by the brief appearances of visitor instrumentation: these included, in 1996, a low-resolution optical spectrograph provided by PSI and the Arcetri (Italy) near-infrared camera, and from late 2002 through 2003, CorMASS, a low-resolution infrared slit spectrograph.

The original $2 \mathrm{~K} \mathrm{CCD}$ camera was replaced in 2007 by a $4 \mathrm{~K}$ imager, which was capable of covering the whole 12 arcminute telescope field of view, courtesy of ITL. In 2009, the National University of Ireland Galway located its ultra-fast imager (GUFI) at VATT; the agreement with NUI Galway continues to the present. In the fall of 2010, after a development time nearly comparable with the history of VATT, a medium-resolution, optical, slit spectrograph, VATTspec, was installed. In 2018, the original $4 \mathrm{~K}$ imager was replaced by a new imager chip of similar dimensions from ITL.

In the twenty-five years since first light, the telescope has had several major upgrades. In December 1998, the Vatican Observatory Foundation was awarded a Science Initiative Grant by The Kresge Foundation; the engineering projects, completed under this grant in 2000, significantly improved the performance of VATT, most obviously in its imaging. In the summer of 2008 , came the installation of new networking and computing equipment, provided through a grant from Hewlett Packard. As a result, the stability of the telescope's networking, and so the efficiency of observations, increased enormously. At the present time, the VATT is undergoing its most significant upgrade since its first light, as described below.

An idea of the scope of scientific projects pursued at VATT can be obtained by looking at the schedules for observers, which are preserved at the VATT website since 1998. These projects cover the whole range of astronomy, from solar system, galactic, and extragalactic, to cosmological astronomy. The members of the Specola have who have observed at the vatT include Boyle, Brown, Consolmagno, Corbally, Funes, Gabor, Kikwaya Eluo, and Omizzolo. In addition, Jonathan Stott, S.J. served his regency with the Specola from 2012 to 2014. A specialist in optical sciences, he worked toward characterizing the optical system of the VATT and also produced a number of striking astro-images with its camera.

The VATT is now in a time of transition. VATT's science in the next ten years will be shaped by two imminent and significant changes. First, the VATT is becoming a robotic telescope, capable of being operated remotely without a human presence necessary at the mountaintop. This involves intensive upgrading of virtually all of the telescope's infrastructure; much of this work has been funded by a generous grant from the Papal Foundation. And second, this 
robotization is coordinated with that for the Steward Observatory's other medium-sized telescopes, the 9o-inch Bok telescope on Kitt Peak and the 61-inch LPL telescope on Mt. Bigelow. When all three are robotic, with each telescope employing a different instrument, simultaneous observations in different modes for the same program will be possible. This will make the three telescopes into a powerful and unique consortium: Arizona Telescope Network (ATN).

The multi-telescope projects involving the Specola for when vaTT becomes a member of the ATN are likely to be near-Earth objects and their physical characteristics, rotational mapping of objects such as NEOs, KBOs, brown dwarfs, and exoplanets, mapping the local galactic neighborhood in ${ }_{3} \mathrm{D}$, and follow-up of asteroseimologically interesting objects in the Kepler and Plato fields.

\section{8}

\section{Conclusion}

In 2014, Funes as director of the Specola prepared a document that outlined the goals and plan of action for the next ten years of the observatory. Much of what has been presented here is based on material from that document. But perhaps the most important part of that document was the vision that Funes presented in its preface. He wrote:

At a colloquium sponsored by the Vatican Observatory on the occasion of the International Year of Astronomy in 2009, Pope Benedict XVI invited those in attendance "to consider the immense progress of scientific knowledge in the modern age and, in a particular way, to turn our gaze anew to the heavens in a spirit of wonder, contemplation and commitment to the pursuit of truth, wherever it is to be found." Inspired by these words and the recent encouragement of Pope Francis to go to the "existential outskirts," and aware of the swift progress of our understanding of the universe, the Vatican Observatory staff strives to address the big questions of astrophysics and cosmology.

We are enthusiastic about our mission. Like all astronomers our deepest desire is to be on the frontier of astronomical research; we share with our colleagues the same excitement in seeking answers to the fundamental questions about the universe: Are we alone? Are there other Earths? How do stars and planets form and evolve? How do galaxies form and evolve? 
What is dark matter and dark energy? What do we know about the universe in its first instants? Are there many universes?

But more fundamentally, our only goal is to do good science; our only commitment is to pursue truth, wherever it is to be found.

In this way, Funes described perfectly the mission of the Vatican Observatory. It has formed not only the science of what we have done over the past several decades but indeed since our founding in 1891 . And it is my fervent hope, as the successor to Funes, that this pursuit of truth will continue to guide our activities for the foreseeable future. 